\title{
A Study on Isomorphic Properties of Circulant Graphs
}

\author{
V. Vilfred Kamalappan ${ }^{\star}$ \\ Department of Mathematics, Central University of Kerala Periye, Kasaragod, Kerala, 671316 India
}

\begin{tabular}{l} 
A R T I C L E I N F O \\
\hline Article history: \\
Received: 13 October, 2017 \\
Accepted: 06 November, 2017 \\
Online: 20 December, 2017 \\
\hline Keywords: \\
Self-Complementary circulant \\
graphs \\
Type-1 and Type-2 isomorphic \\
circulant graphs \\
Cartesian product and factor- \\
ization \\
prime and composite circulant \\
graphs \\
Factorization theorem, Funda- \\
mental theorem
\end{tabular}

\section{Introduction}

This paper is an extension of work originally presented in ICMSAO2017 [1] and covers the author's study on a few isomorphic properties of circulant graphs that includes (i) Existence of selfcomplementary circulant graphs; (ii) Type-2 isomorphism, a new type of isomorphism other than already known Adam's isomorphism of circulant graphs that helps to obtain graphs without CI-property and abelian groups and (iii) Cartesian product and factorization of circulant graphs similar to the theory of product and factorization of natural numbers.

Beauty comes out of symmetry as well as asymmetry. Investigation of symmetries/asymmetries of structures yield powerful results in Mathematics. Circulant graphs form a class of highly symmetric mathematical (graphical) structures. In 1846 Catalan (cf. [2]) introduced circulant matrices and properties of circulant graphs have been investigated by many authors [1-20]. An excellent account of circulant matrices can be found in the book by Davis [2] and circulant graphs in the article [11].

If a graph $G$ is circulant, then its adjacency matrix $A(G)$ is circulant. It follows that if the first row of the adjacency matrix of a circulant graph is $\left[a_{1}, a_{2}, \ldots, a_{n}\right]$, then $a_{1}=0$ and $a_{i}=a_{n-i+2}, 2 \leq i \leq n[15,17]$.

Through-out this paper, for a set $R=\left\{r_{1}, r_{2}, \ldots, r_{k}\right\}$,
$C_{n}(R)$ denotes circulant graph $C_{n}\left(r_{1}, r_{2}, \ldots, r_{k}\right)$ where $1 \leq r_{1}<r_{2}<\ldots<r_{k} \leq\left[\frac{n}{2}\right]$. Only connected circulant graphs of finite order are considered, $V\left(C_{n}(R)\right)$ $=\left\{v_{0}, v_{1}, v_{2}, \ldots, v_{n-1}\right\}$ with $v_{i}$ adjacent to $v_{i+r}$ for each $r \in R$, subscript addition taken modulo $n$ and all cycles have length at least 3 , unless otherwise specified, $0 \leq i \leq n-1$. However when $\frac{n}{2} \in R$, edge $v_{i} v_{i+\frac{n}{2}}$ is taken as a single edge for considering the degree of the vertex $v_{i}$ or $v_{i+\frac{n}{2}}$ and as a double edge while counting the number of edges or cycles in $C_{n}(R), 0 \leq i \leq n-1$. Generally, write $C_{n}$ for $C_{n}(1)$ and $C_{n}\left(1,2, \ldots,\left\lfloor\frac{n}{2}\right\rfloor\right)$ for $K_{n}$. We will often assume, with-out further comment, that the vertices are the corners of a regular $n-g o n$, labeled clockwise. Circulant graphs $C_{16}(1,2,7), C_{16}(2,3,5)$, $C_{27}(1,3,8,10), C_{27}(3,4,5,13)$ and $C_{27}(2,3,7,11)$ are shown in Figures 1 - 5. Now, let us consider the following definitions and results that are useful in the subsequent sections.

Definition 1.1. [17] Let $n$ and $r$ be positive integers with $n \geq 4$ and $r<\frac{n}{2}$. Then, clearly, $C_{n}(r)$ consists of $a$ collection of cycles $\left(v_{0} v_{r} v_{2 r} \ldots v_{0}\right),\left(v_{1} v_{1+r} v_{1+2 r} \ldots v_{1}\right), \ldots$, $\left(v_{r-1} v_{2 r-1} v_{3 r-1} \ldots v_{r-1}\right)$. If $d=\operatorname{gcd}(n, r)$, then there are $d$ such disjoint cycles, each of length $\frac{n}{d}$. We say that each of these cycles is of period $r$, length $\frac{n}{d}$ and rotation $\frac{r}{d}$.

If $r=\frac{n}{2}$, then obviously $C_{n}(r)$ is just a $1-$ factor. Since $C_{n}(R)$ is just the union of the cycles $C_{n}(r)$ for $r \in R$, we have a decomposition of $C_{n}(R)$.

${ }^{*}$ Corresponding Author: V. Vilfred Kamalappan Email: vilfredkamalv@cukerala.ac.in, Contact No. 00919443577502 

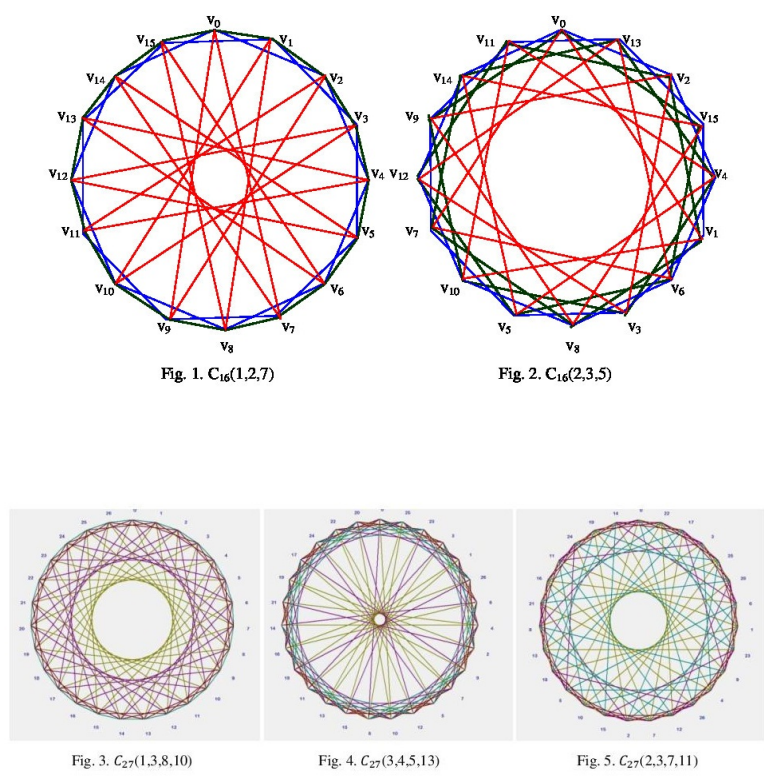

Theorem 1.2. [17] Let $r \in R$. Then, in $C_{n}(R)$, the length of a cycle of period $r$ is $\frac{n}{\operatorname{gcd}(n, r)}$ and the number of disjoint periodic cycles of period $r$ is $\operatorname{gcd}(n, r)$.

Corollary 1.3. [17] In $C_{n}(R)$, the length of a cycle of period $r$ is $n$ if and only if $\operatorname{gcd}(n, r)=1, r \in R$.

Remark 1.4. [17] Let $|R|=k$. Then, the circulant graph $C_{n}(R)$ for a set $R=\left\{r_{1}, r_{2}, \ldots, r_{k}\right\}$ is $(2 k-1)$-regular if $\frac{n}{2} \in R$ and $2 k$-regular otherwise. $\square$

The following Lemmas are useful to obtain one-toone mappings.

Lemma 1.5. [15] Let $A$ and $B$ be two non-empty sets. Let $f: A \rightarrow B$ be a mapping. Then, $f$ is one-to-one if and only if $f /_{A^{\prime}}$ is one-to-one for every non-empty subset $A^{\prime}$ of $A$.

Lemma 1.6. [15] Let $A$ and $B$ be non-empty sets and $A_{1}, A_{2}, \ldots, A_{k}$ be a partition of $A$ (each $A_{i}$ being nonempty, $i=1,2, \ldots, k)$. Let $f: A \rightarrow B$ be a mapping. Then $f$ is one-to-one if and only if $f A_{A_{i}}$ is one-to-one for every $i, i=1,2, \ldots, k$. $\square$

\section{On self-complementary circu- lant graphs}

In 1962, Horst Sachs [14] proved that the sufficient condition for the existence of a self-complementary circulant graph of order $n$ is that every prime factor $p$ of $n$ should satisfy $p \equiv 1(\bmod 4)$. He also conjectured that the sufficient condition is a necessary one. We have proved that the self-complementary circulant graph on $n$ vertices does not exist if $n$ has any prime factor which is not of the form $4 m+1, m \in \mathbb{N}$. Thereby, we establish that the sufficient condition is also a necessary one. The proof is based on counting the number of disjoint cycles of a particular length in $K_{n}$ and is given in this section $[4,17]$. Graphs given in Figures 6 and 7 are self-complementary but not of circulant whereas graphs given in Figures 8 and 9 are self-complementary circulant graphs.
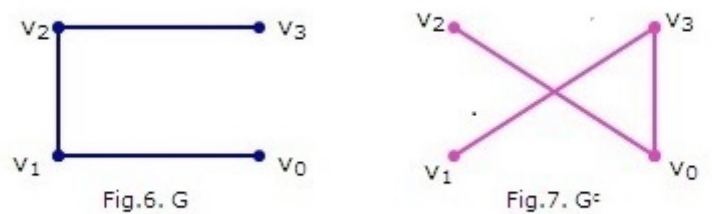

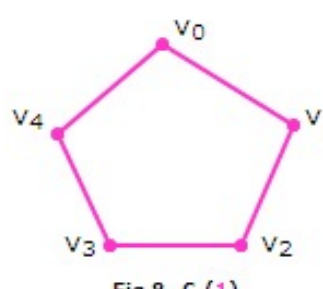

Fig.8. Cs(1)

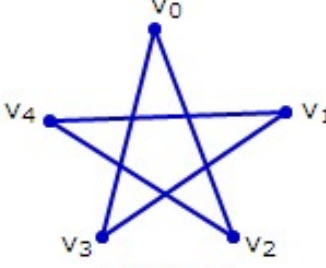

Fig.9. $C_{s}(2)$
Theorem 2.1. [17] If $C_{n}(R) \cong C_{n}(S)$, then there is a bijection $f$ from $R$ to $S$ so that for all $r \in R, \operatorname{gcd}(n, r)=$ $\operatorname{gcd}(n, f(r))$.

Proof. The proof is by induction on the order of $R$.

Theorem 2.2. [9] If graph $G$ of order $n$ is selfcomplementary, then $n \equiv 0,1(\bmod 4)$. $\square$

Theorem 2.3. [17] If $C_{n}(R)$ for a set $R=\left\{r_{1}, r_{2}, \ldots, r_{k}\right\}$ is self-complementary, then $n \equiv 1(\bmod 4)$.

Proof. Using Theorem 2.2 we get $n \equiv 0,1(\bmod 4)$. When $n$ is even, a circulant graph is of even degree if and only if its complement is of odd degree since any circulant graph is a regular graph. Thus, selfcomplementary circulant graph of even order doesn't exist. Hence, we get the result.

Theorem 2.4. [17] If $C_{n}(R)$ for a set $R=\left\{r_{1}, r_{2}, \ldots, r_{k}\right\}$ is self-complementary, then $n=4 m+1, k=m$ and $\left|S_{i}\right|$ is even where $S_{i}=\left\{j: \operatorname{gcd}(n, j)=\operatorname{gcd}(n, i), 1 \leq j \leq\left[\frac{n}{2}\right]\right\}$, for all $i, i=1,2, \ldots,\left[\frac{n}{2}\right]$.

Proof. Using Theorem 2.3, we get, $n=4 m+1$. This implies that the degree (of each vertex) of a selfcomplementary circulant graph of order $4 m+1$ is $2 m$ which implies $k=m$.

Let $C_{4 m+1}(R)$ be a self-complementary circulant graph for $R=\left\{r_{1}, r_{2}, \ldots, r_{m}\right\}$. If it contains a cycle of period $r$ (and of length $\frac{n}{\operatorname{gcd}(n, r)}$, using Theorem 1.2 , then its complement also contains a periodic cycle of period, say, $s$ such that $\frac{n}{\operatorname{gcd}(n, s)}=\frac{n}{\operatorname{gcd}(n, r)}$, $1 \leq r, s \leq\left[\frac{n}{2}\right]$, using Theorem 1.2 . This implies that $\operatorname{gcd}(n, s)=\operatorname{gcd}(n, r)$. Here we consider that the cycles of periods $r_{i}$ and $n-r_{i}$ are the same in $C_{n}\left(r_{1}, r_{2}, \ldots, r_{k}\right)$, $1 \leq i \leq k$. Combining the above arguments, we get the result. 
Remark 2.5. The above theorem states that if a selfcomplementary circulant graph of order $n$ exists, then $n=4 m+1$ and so it is $2 m$-regular and the number of periodic cycles of length $i$ in $K_{n}$ is always even for each $i$, $1 \leq i \leq\left[\frac{n}{2}\right]$.

Theorem 2.6. [17] Self-complementary circulant graph of order $n$ doesn't exist when $n$ has any prime factor of the form $2(2 m-1)+1, m \in \mathbb{N}$.

Proof. Using Theorem 2.3, $n=4 a+1, a+1 \in \mathbb{N}$.

Let $n=p_{1}^{n_{1}} p_{2}^{n_{2}} \ldots p_{j}^{n_{j}}$ where $p_{1}, p_{2}, \ldots, p_{j}$ are the (odd) prime factors of $n$. Let $p_{i} \neq 4 m+1$ for at least one $i, 1 \leq i \leq j$ and for any $m \in \mathbb{N}$. This implies, $p_{i}=2(2 q-1)+1$ for some $q \in \mathbb{N}$. Consider the circulant graph $C_{4 a+1}(1,2, \ldots, 2 a) \cong K_{4 a+1}=K_{n}$. Let $r$ be the natural number such that $r=\frac{n}{p_{i}}$.

Aim To find out all natural numbers lying between 1 and $n$ such that g.c.d. of each one of them with $n$ is exactly $r$.

Let $\operatorname{gcd}(n, p r+s)=r$ where $p, s+1 \in \mathbb{N}$ such that $0 \leq s<r$ and $1 \leq p r+s \leq n=r p_{i}$. This implies that $s=0$ and so $\operatorname{gcd}(n, r p+s)=\operatorname{gcd}(n, r p)=\operatorname{gcd}\left(r p_{i}, r p\right)=r$ where $1 \leq p r \leq p_{i} r=n$. Thus we $\operatorname{get} \operatorname{gcd}\left(p, p_{i}\right)=1$ where $p \leq p_{i}$ which is greater than 1 . Therefore the possible values of $p$ are $1,2, \ldots, p_{i}-1$.

Thus $r, 2 r, 3 r, \ldots,\left(p_{i}-1\right) r$ are the only numbers lying between 1 and $n$ such that g.c.d. of $n$ with each one of them is exactly $r$. This implies $r, 2 r, 3 r, \ldots,\left(p_{i}-1\right) r$ are the possible periods of cycles of length $p_{i}$ each, in $C_{n}\left(1,2, \ldots,\left[\frac{n}{2}\right]\right)$ since the length of a cycle of period $r p$ in $C_{n}\left(1,2, \ldots,\left[\frac{n}{2}\right]\right)$ is $\frac{n}{\operatorname{gcd}(n, r p)}=\frac{r p_{i}}{\operatorname{gcd}\left(r p_{i}, r p\right)}=\frac{p_{i}}{\operatorname{gcd}\left(p_{i}, p\right)}=p_{i}$ for $p=1,2, \ldots, p_{i}-1$, using Theorem 1.2 .

In $C_{n}\left(1,2, \ldots,\left[\frac{n}{2}\right]\right)$,

the cycles of period $r$ and $n-r\left(=\left(p_{i}-1\right) r\right)$ are the same,

the cycles of period $2 r$ and $\left(p_{i}-2\right) r$ are the same,

the cycles of period $\frac{\left(p_{i}-1\right) r}{2}$ and $\frac{\left(p_{i}+1\right) r}{2}$ are the same.

Thus, there are $\frac{p_{i}-1}{2}$ number of possible distinct periodic cycles of (periods $r, 2 r, \ldots, \frac{p_{i}-1}{2}$ and) length $p_{i}$, each in $C_{n}\left(1,2, \ldots,\left[\frac{n}{2}\right]\right)$.

Now, $\frac{p_{i}-1}{2}=2 q-1$ is an odd number. This implies, any circulant graph of order $n$ and its complementary circulant graph contain unequal number of periodic cycles of length $p_{i}$, each. This implies that self-complementary circulant graph of order $n$ does n't exist when $n$ contains any prime factor of the form $2(2 q-1)+1, q \in \mathbb{N}$, by Remark 2.5 .

Thus, when $n=9,21,33,49,57,69,77,81,93$, etc., self-complementary circulant graph doesn't exist on $n$ vertices, by Theorem 2.6, even though in each case, $n \equiv 1(\bmod 4)$.

Now, by combining Theorem 2.6 and the sufficient condition for the existence of a self-complementary circulant graph of order $n$, we get the following result.

Theorem 2.7. [17] The necessary and sufficient condition for the existence of a self-complementary circulant graph of order $n$ is that each prime factor $p$ of $n$ should satisfy $p \equiv 1(\bmod 4)$.

\section{On Isomorphism of Circulant Graphs}

In this section, Type-2 isomorphism, a new type of isomorphism different from already known Adam's isomorphism of circulant graphs, main results related to it and families of new abelian groups obtained from isomorphic circulant graphs are presented. Type-2 isomorphic circulant graphs have the property that they are isomorphic graphs without Cayley Isomorphism (CI) property.

Definition 3.1. [12] A circulant graph $C_{n}(R)$ is said to have the CI-property if whenever $C_{n}(S)$ is isomorphic to $C_{n}(R)$, there is some $a \in \mathbb{Z}_{n}^{*}$ for which $S=a R$.

Lemma 3.2. [16] Let $S$ be a non-empty subset of $\mathbb{Z}_{n}$ and $x \in \mathbb{Z}_{n}$. Define a mapping $\Phi_{n, x}: S \rightarrow \mathbb{Z}_{n}$ such that $\Phi_{n, x}(s)=x s$ for every $s \in S$ under multiplication modulo $n$. Then, $\Phi_{n, x}$ is bijective if and only if $S=\mathbb{Z}_{n}$ and $\operatorname{gcd}(n, x)=1$.

Definition 3.3. [3] Circulant graphs $C_{n}(R)$ and $C_{n}(S)$ for $R=\left\{r_{1}, r_{2}, \ldots, r_{k}\right\}$ and $S=\left\{s_{1}, s_{2}, \ldots, s_{k}\right\}$ are Adam's isomorphic if there exists a positive integer $x$ relatively prime to $n$ with $S=\left\{x r_{1}, x r_{2}, \ldots, x r_{k}\right\}_{n}^{*}$ where $<r_{i}>_{n}^{*}$, the reflexive modular reduction of a sequence $\left\langle r_{i}>\right.$ is the sequence obtained by reducing each $r_{i}$ modulo $n$ to yield $r_{i}^{\prime}$ and then replacing all resulting terms $r_{i}^{\prime}$ which are larger than $\frac{n}{2}$ by $n-r_{i}^{\prime}$.

Lemma 3.4. [16] Let $m, r, t \in \mathbb{Z}_{n} \in \operatorname{gcd}(n, r)=m>1$ and $0 \leq t \leq \frac{n}{m}-1$. Then the mapping $\Theta_{n, r, t}: \mathbb{Z}_{n} \rightarrow \mathbb{Z}_{n}$ defined by $\Theta_{n, r, t}(x)=x+j$ tm for every $x \in \mathbb{Z}_{n}$ under arithmetic modulo $n$ is bijective where $x=j+q m, 0 \leq j \leq m-1$, $0 \leq q \leq \frac{n}{m}-1$ and $j, q \in \mathbb{Z}_{n}$. $\square$

Theorem 3.5. [16] Let $V\left(C_{n}(R)\right)=\left\{v_{0}, v_{1}, \ldots, v_{n-1}\right\}$, $V\left(K_{n}\right)=\left\{u_{0}, u_{1}, \ldots, u_{n-1}\right\}, r \in R$ and $\operatorname{gcd}(n, r)=m>$ 1. Then the mapping $\Theta_{n, r, t}: V\left(C_{n}(R)\right) \rightarrow V\left(K_{n}\right)$ defined by $\Theta_{n, r, t}\left(v_{x}\right)=u_{x+j t m}$ and $\Theta_{n, r, t}\left(\left(v_{x}, v_{x+s}\right)\right)=$ $\left(\Theta_{n, r, t}\left(v_{x}\right), \Theta_{n, r, t}\left(v_{x+s}\right)\right)$ for every $x \in \mathbb{Z}_{n}, x=j+q m$, $0 \leq j \leq m-1,0 \leq q, t \leq \frac{n}{m}-1$ and $s \in R$, under subscript arithmetic modulo $n$, for a set $R=\left\{r_{1}, r_{2}, \ldots, r_{k}\right.$, $\left.n-r_{k}, n-r_{k-1}, \ldots, n-r_{1}\right\}$ is one-to-one, preserves adjacency and $\Theta_{n, r, t}\left(C_{n}(R)\right) \cong C_{n}(R)$ for $t=0,1,2, \ldots, \frac{n}{m}-1$.

Definition 3.6. [16] For a given $C_{n}(R)$ and for a particular value of $t, 0 \leq t \leq \frac{n}{m}-1$, if $\Theta_{n, r, t}\left(C_{n}(R)\right)=C_{n}(S)$ for some $S \subseteq\left[1, \frac{n}{2}\right]$ and $S \neq x R$ for all $x \in \Phi_{n}$ under reflexive modulo $n$, then $C_{n}(R)$ and $C_{n}(S)$ are called Type-2 isomorphic circulant graphs w.r.t. $r, r \in R$. In this case, subsets $R$ and $S$ of $\mathbb{Z}_{n}$ are called Type-2 isomorphic subsets of $\mathbb{Z}_{n}$ w.r.t.r.

Clearly, Type-2 isomorphic circulant graphs are circulant graphs without CI-property. We obtained the following results on Type- 2 isomorphism. 
Theorem 3.7. [16] For $n \geq 2, k \geq 3,1 \leq 2 s-1 \leq 2 n-1$, $n \neq 2 s-1, R=\left\{2 s-1,4 n-2 s+1,2 p_{1}, 2 p_{2}, \ldots, 2 p_{k-2}\right\}$ and $S=\left\{2 n-(2 s-1), 2 n+2 s-1,2 p_{1}, 2 p_{2}, \ldots, 2 p_{k-2}\right\}$, circulant graphs $C_{8 n}(R)$ and $C_{8 n}(S)$ are Type-2 isomorphic (and without $C I-$ property) where $\operatorname{gcd}\left(p_{1}, p_{2}, \ldots, p_{k-2}\right)$ $=1$ and $n, s, p_{1}, p_{2}, \ldots, p_{k-2} \in \mathbb{N}$.

Theorem 3.8. [16] For $R=\left\{2 r-1,2 s-1,2 p_{1}, 2 p_{2}, \ldots\right.$, $\left.2 p_{k-2}\right\}, n \geq 2, k \geq 3,1 \leq t \leq\left[\frac{n}{2}\right], 1 \leq 2 r-1<2 s-1 \leq\left[\frac{n}{2}\right]$, $\operatorname{gcd}\left(p_{1}, p_{2}, \ldots, p_{k-2}\right)=1$ and $n, r, s, t, p_{1}, p_{2}, \ldots, p_{k-2} \in \mathbb{N}$, if $\Theta_{n, 2, t}\left(C_{n}(R)\right)$ and $C_{n}(R)$ are Type-2 isomorphic circulant graphs for some $t$, then $n \equiv 0(\bmod 8), 2 r-1+2 s-1$ $=\frac{n}{2}, t=\frac{n}{8}$ or $\frac{3 n}{8}, 2 r-1 \neq \frac{n}{8}, 1 \leq 2 r-1 \leq \frac{n}{4}$ and $n \geq 16$.

Definition 3.9. [1] Let $A d_{n}=\left\{\Phi_{n, x}: x \in \Phi_{n}\right\}$, $A d_{n}(R)=\left\{\Phi_{n, x}(R): x \in \Phi_{n}\right\}=\left\{x R: x \in \Phi_{n}\right\}$ and $A d_{n}\left(C_{n}(R)\right)=T 1_{n}\left(C_{n}(R)\right)=\left\{\Phi_{n, x}\left(C_{n}(R)\right): x \in \Phi_{n}\right\}=$ $\left\{C_{n}(x R): x \in \Phi_{n}\right\}$ for a set $R=\left\{r_{1}, r_{2}, \ldots, r_{k}, n-r_{k}, n-\right.$ $\left.r_{k-1}, \ldots, n-r_{1}\right\} \subseteq \mathbb{Z}_{n}$. Define 'o' in $A d_{n}\left(C_{n}(R)\right)$ such that $\Phi_{n, x}\left(C_{n}(R)\right) \circ \Phi_{n, y}\left(C_{n}(R)\right)=\Phi_{n, x y}\left(C_{n}(R)\right)$ and $C_{n}(x R)$ ○ $C_{n}(y R)=C_{n}((x y) R)$ for every $x, y \in \Phi_{n}$, under arithmetic modulo $n$. Clearly, $A d_{n}\left(C_{n}(R)\right)$ is the set of all circulant graphs that are Adam's isomorphic to $C_{n}(R)$ and $\left(A d_{n}\left(C_{n}(R)\right), \circ\right)=\left(T 1_{n}\left(C_{n}(R)\right)\right.$, o $)$ is an abelian group and we call it as the Adam's group or Type-1 group on $C_{n}(R)$ under 'o'.

Definition 3.10. [1] Let $V\left(C_{n}(R)\right)=\left\{v_{0}, v_{1}, \ldots, v_{n-1}\right\}$, $V\left(K_{n}\right)=\left\{u_{0}, u_{1}, \ldots, u_{n-1}\right\}, r \in R, m, q, t, t^{\prime}, x \in \mathbb{Z}_{n}$ such that $\operatorname{gcd}(n, r)=m>1, x=j+q m, 0 \leq j \leq$ $m-1$ and $0 \leq q, t, t^{\prime} \leq \frac{n}{m}-1$. Define $\Theta_{n, r, t}: \mathbb{Z}_{n} \rightarrow$ $\mathbb{Z}_{n}$ and $\Theta_{n, r, t}: V\left(C_{n}(R)\right) \rightarrow V\left(K_{n}\right)$ such that $\Theta_{n, r, t}(x)$ $=x+j t m, \Theta_{n, r, t}\left(v_{x}\right)=u_{x+j t m}$ and $\Theta_{n, r, t}\left(\left(v_{x}, v_{x+s}\right)\right)=$ $\left(\Theta_{n, r, t}\left(v_{x}\right), \Theta_{n, r, t}\left(v_{x+s}\right)\right)$ for every $x \in \mathbb{Z}_{n}$ and $s \in R$, under subscript arithmetic modulo n. Let $s \in \mathbb{Z}_{n}, V_{n, r}=\left\{\Theta_{n, r, t}\right.$ : $\left.t=0,1, \ldots, \frac{n}{m}-1\right\}, V_{n, r}(s)=\left\{\Theta_{n, r, t}(s): t=0,1, \ldots, \frac{n}{m}-1\right\}$ and $V_{n, r}\left(C_{n}(R)\right)=\left\{\Theta_{n, r, t}\left(C_{n}(R)\right): t=0,1, \ldots, \frac{n}{m}-1\right\}$. Define 'o' in $V_{n, r}$ such that $\Theta_{n, r, t} \circ \Theta_{n, r, t^{\prime}}=\Theta_{n, r, t+t^{\prime}}$, $\left(\Theta_{n, r, t} \circ \Theta_{n, r, t^{\prime}}\right)(x)\left(=\Theta_{n, r, t}\left(\Theta_{n, r, t^{\prime}}(x)\right)=\Theta_{n, r, t}\left(x+j t^{\prime} m\right)\right.$ $\left.=\left(x+j t^{\prime} m\right)+j t m=x+j\left(t+t^{\prime}\right) m\right)=\Theta_{n, r, t+t^{\prime}}(x)$ and $\Theta_{n, r, t}\left(C_{n}(R)\right) \circ \Theta_{n, r, t^{\prime}}\left(C_{n}(R)\right)=\Theta_{n, r, t+t^{\prime}}\left(C_{n}(R)\right)$ for every $\Theta_{n, r, t}, \Theta_{n, r, t^{\prime}} \in V_{n, r}$ where $t+t^{\prime}$ is calculated under add $i$ tion modulo $\frac{n}{m}$. Clearly, $\left(V_{n, r}(s)\right.$, o $)$ and $\left(V_{n, r}\left(C_{n}(R)\right)\right.$, o) are abelian groups for every $s \in \mathbb{Z}_{n}$.

$V_{n, r}\left(C_{n}(R)\right)$ contains all isomorphic circulant graphs of Type-2 of $C_{n}(R)$ w.r.t. $r$, if exist. Let $T 2_{n, r}\left(C_{n}(R)\right)=\left\{C_{n}(R)\right\} \cup\left\{C_{n}(S): C_{n}(S)\right.$ is Type-2 isomorphic to $C_{n}(R)$ w.r.t. $\left.r\right\}$. Thus, $T 2_{n, r}\left(C_{n}(R)\right)=$ $\left\{C_{n}(R)\right\} \cup\left\{\Theta_{n, r, t}\left(C_{n}(R)\right): \Theta_{n, r, t}\left(C_{n}(R)\right)=C_{n}(S)\right.$ and $C_{n}(S)$ is Type-2 isomorphic to $C_{n}(R)$ w.r.t. $r, 0 \leq t \leq$ $\left.\frac{n}{m}-1\right\}=\left\{\Theta_{n, r, 0}\left(C_{n}(R)\right)\right\} \cup\left\{\Theta_{n, r, t}\left(C_{n}(R)\right): \Theta_{n, r, t}\left(C_{n}(R)\right)\right.$ $=C_{n}(S)$ and $C_{n}(S)$ is Type-2 isomorphic to $C_{n}(R)$, $\left.0 \leq t \leq \frac{n}{m}-1\right\} \subseteq V_{n, r}\left(C_{n}(R)\right)$ and $\left(T 2_{n, r}\left(C_{n}(R)\right)\right.$, o $)$ is a subgroup of $\left(V_{n, r}\left(C_{n}(R)\right)\right.$, o). Clearly, $T 1_{n}\left(C_{n}(R)\right) \cap$ $T 2_{n, r}\left(C_{n}(R)\right)=\left\{C_{n}(R)\right\}$. And $C_{n}(R)$ has Type-2 isomorphic circulant graph w.r.t. $r$ if and only if $T 2_{n, r}\left(C_{n}(R)\right)$ $\neq\left\{C_{n}(R)\right\}$ if and only if $T 2_{n, r}\left(C_{n}(R)\right) \cap\left\{C_{n}(R)\right\} \neq \Phi$ if and only if $\left|T 2_{n, r}\left(C_{n}(R)\right)\right|>1[1]$.

Definition 3.11. [1] For any circulant graph $C_{n}(R)$, if $T 2_{n, r}\left(C_{n}(R)\right) \neq\left\{C_{n}(R)\right\}$, then $\left(T 2_{n, r}\left(C_{n}(R)\right)\right.$, o $)$ is called the Type-2 group of $C_{n}(R)$ w.r.t. $r$ under ' $\mathrm{o}$.
Theorem 3.12. [1] Let $p$ be an odd prime and $k \geq 3$. Then, for $i=1$ to $p, d_{i}=(i-1) n p+1$ and $R_{i}=\left\{d_{i}\right.$, $n p^{2}-d_{i}, n p^{2}+d_{i}, 2 n p^{2}-d_{i}, 2 n p^{2}+d_{i}, 3 n p^{2}-d_{i}, 3 n p^{2}+$ $d_{i}, \ldots,(p-1) n p^{2}-d_{i},(p-1) n p^{2}+d_{i}, n p^{3}-d_{i}, p p_{1}, p p_{2}, \ldots$, $\left.p p_{k-2}, p\left(n p^{3}-p_{k-2}\right), p\left(n p^{3}-p_{k-3}\right), \ldots, p\left(n p^{3}-p_{1}\right)\right\}$, circulant graphs $C_{n p^{3}}\left(R_{i}\right)$ are Type-2 isomorphic (and without CI-property) where $g c d\left(p_{1}, p_{2}, \ldots, p_{k-2}\right)=1$ and $n, p_{1}, p_{2}, \ldots, p_{k-2} \in \mathbb{N}$.

Theorem 3.13. [1] Let $p$ be an odd prime, $k \geq 3$, $1 \leq i \leq p, d_{i}=(i-1) n p+1, R_{i}=\left\{d_{i}, n p^{2}-d_{i}\right.$, $n p^{2}+d_{i}, 2 n p^{2}-d_{i}, 2 n p^{2}+d_{i}, 3 n p^{2}-d_{i}, 3 n p^{2}+d_{i}$, $\ldots,(p-1) n p^{2}-d_{i},(p-1) n p^{2}+d_{i}, n p^{3}-d_{i}, p p_{1}, p p_{2}$, $\left.\ldots, p p_{k-2}, p\left(n p^{3}-p_{k-2}\right), p\left(n p^{3}-p_{k-3}\right), \ldots, p\left(n p^{3}-p_{1}\right)\right\}$, $T 2\left(R_{i}\right)=\left\{\Theta_{n p^{3}, p, j n}\left(R_{i}\right): j=1,2, \ldots, p\right\}, T 2\left(C_{n p^{3}}\left(R_{i}\right)\right)=$ $\left\{\Theta_{n p^{3}, p, j n}\left(C_{n p^{3}}\left(R_{i}\right)\right): j=1,2, \ldots, p\right\}, \operatorname{gcd}\left(p_{1}, p_{2}, \ldots, p_{k-2}\right)$ $=1$ and $n, p_{1}, p_{2}, \ldots, p_{k-2} \in \mathbb{N}$. Then $T 2_{n p^{3}, p}\left(R_{i}\right)$ $=T 2\left(R_{j}\right), T 2_{n p^{3}, p}\left(C_{n p^{3}}\left(R_{i}\right)\right)=T 2\left(C_{n p^{3}}\left(R_{j}\right)\right)$ and $\left(V_{n p^{3}, p}\left(R_{i}\right), \circ\right),\left(V_{n p^{3}, p}\left(C_{n p^{3}}\left(R_{i}\right)\right), \circ\right)$ and $\left(T 2_{n p^{3}, p}\left(R_{i}\right), \circ\right)$ are abelian groups, $1 \leq i, j \leq p$. Moreover, $\left(T 2_{n p^{3}, p}\left(C_{n p^{3}}(\right.\right.$ $\left.R_{i}\right)$ ), o) is the Type-2 group of order $p$ on $C_{n p^{3}}\left(R_{i}\right)$ w.r.t. $r=p, 1 \leq i, j \leq p$.

Circulant graphs $C_{16}(1,2,7)$ and $C_{16}(2,3,5)$ are Type-2 isomorphic and $C_{27}(1,3,8,10), C_{27}(3,4,5,13)$ and $C_{27}(2,3,7,11)$ are also. See Figures $1-5$.

\section{Cartesian Product and Factoriza- tion of Circulant Graphs}

Just as integers can be factored into prime numbers, there are many results on decompositions of structures throughout mathematics [6]. The standard products - Cartesian, lexicographic, tensor, and strong - all belong to a class of products introduced by Imrich and Izbicki and called $B$ - products [8]. In this section, a few important results that are obtained in our study of Cartesian product and factorization of circulant graphs, similar to the theory of product and factorization of natural numbers, are presented (For details see [15]). Graphs $C_{5} \square C_{6}$ and $C_{30}(5,6)$ are isomorphic and are given in Figures 12 and 13 . One can see the difficulty of this study from this example.

Definition 4.1. [9] The cross product or Cartesian product of two simple graphs $G(V, E)$ and $H(W, F)$ is the simple graph $G \square H$ with vertex set $V \times W$ in which two vertices $u=\left(u_{1}, u_{2}\right)$ and $v=\left(v_{1}, v_{2}\right)$ are adjacent if and only if either $u_{1}=v_{1}$ and $u_{2} v_{2} \in F$ or $u_{2}=v_{2}$ and $u_{1} v_{1} \in E$.

Theorem 4.2. [15] Let $G$ be a connected graph of order $n, n>2$. Then, $P_{2} \square G$ is circulant if and only if $G \cong H$ or $P_{2} \square H$ where $H$ is a connected circulant graph of odd order.

Theorem 4.3. [15] Let $G$ be a connected graph of order $n \geq 2$. Then $C_{4} \square G$ is circulant if and only if $G$ is circulant of odd order. $\square$

Theorem 4.4. [15] If $G$ and $H$ are connected graphs and $G \square H$ is circulant, then $G$ and $H$ are circulants. $\square$ 
Graphs $P_{2} \square C_{3}$ and $P_{2} \square C_{4}$ are given in Figures 10 and 11 and $C_{5} \square C_{6}$ and $C_{30}(5,6) \cong C_{5} \square C_{6}$ in 12 and 13 , respectively.

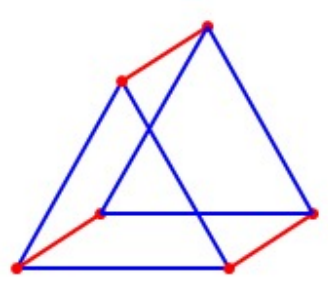

Fig.10. $P_{2} \times C_{3}$

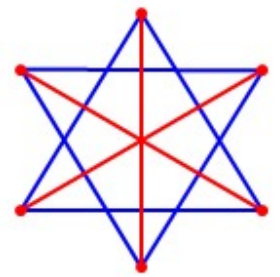

Fig.11. $C_{6}(2,3)$
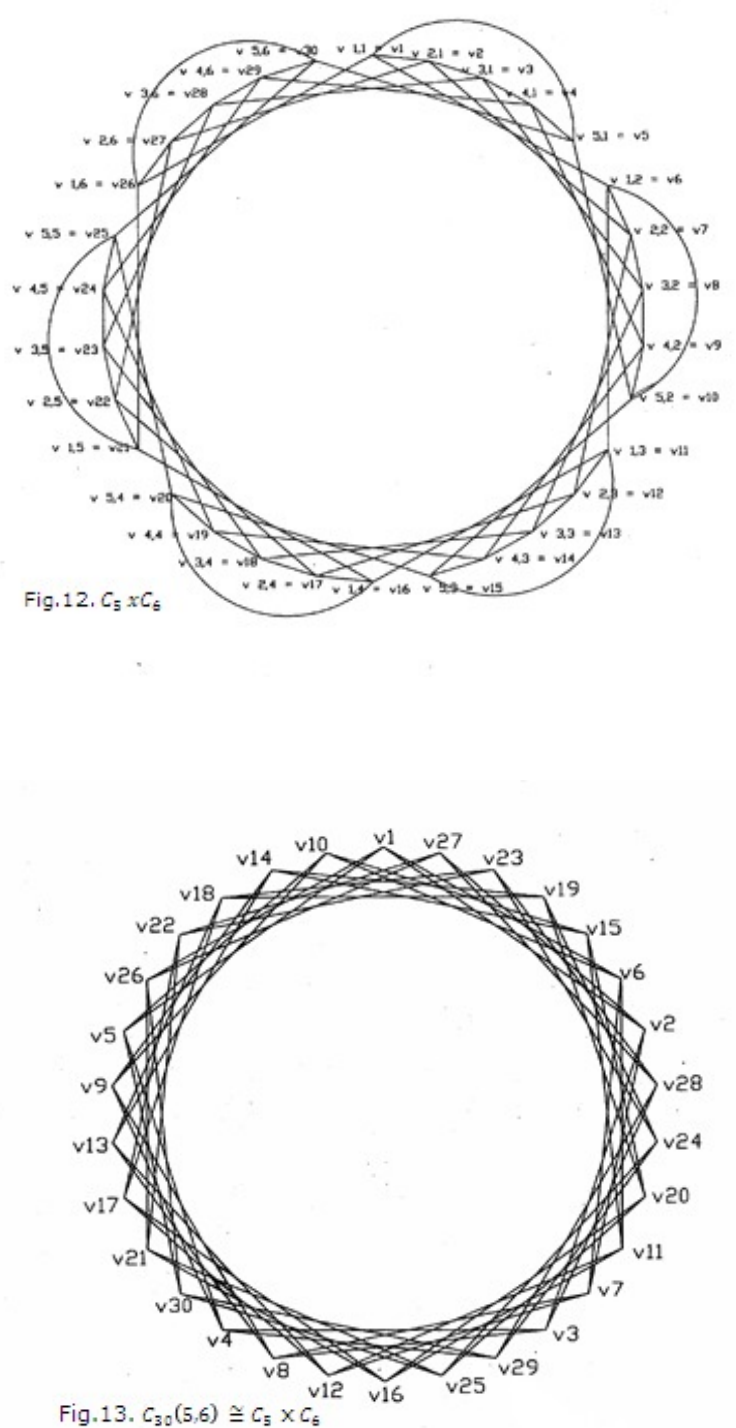

Theorem 4.5. [15] Let $G$ and $H$ be connected graphs, each of order $>2$. Then $G \square H$ is circulant if and only if $G$ and $H$ are circulants and satisfy one of the following conditions:

(i) $G \cong C_{m}(R) ; H \cong C_{n}(S)$ and $\operatorname{gcd}(m, n)=1$. (ii) $G \cong C_{2 m+1}(R) ; H \cong C_{2 n+1}(S), P_{2} \square C_{2 n+1}(S), C_{4} \square$ $C_{2 n+1}(S)$ or $C_{2^{k}(2 n+1)}(S)$ and $g c d\left(2 m+1,2^{k}(2 n+1)\right)$ $=1, k \in \mathbb{N}$.

(iii) $G \cong P_{2} \square C_{2 m+1}(R) ; H \cong C_{2 n+1}(S)$ or $P_{2} \square C_{2 n+1}(S)$ and $\operatorname{gcd}(2 m+1,2 n+1)=1$.

(iv) $G \cong C_{2^{k}(2 m+1)}(R) \neq P_{2} \square C_{2^{k-1}(2 m+1)}(T)$ for any $C_{2^{k-1}(2 m+1)}(T) ; H \cong C_{2 n+1}(S)$ and $\operatorname{gcd}\left(2^{k}(2 m+\right.$ 1), $2 n+1)=1, k \in \mathbb{N}$.

(v) $G \cong C_{4} \square C_{2 m+1}(R) ; H \cong C_{2 n+1}(S)$ and $\operatorname{gcd}(2 m+$ $1,2 n+1)=1$.

(vi) $G \cong C_{2^{k}(2 m+1)}(R) \neq C_{4} \square C_{2^{k-2}(2 m+1}(T), \quad P_{2}$ $\square \quad C_{2^{k-1}(2 m+1)}(U)$ for any $C_{2^{k-2}(2 m+1}(T)$ and $C_{2^{k-1}(2 m+1)}(U) ; H \cong C_{2 n+1}(S)$ and $\operatorname{gcd}\left(2^{k}(2 m+\right.$ 1), $2 n+1)=1, k \in \mathbb{N}, k \geq 2$.

Definition 4.6. [15] A non-trivial graph $G$ is said to be prime if $G=G_{1} \square G_{2}$ implies $G_{1}$ or $G_{2}$ is trivial; $G$ is composite if it is not prime.

Definition 4.7. [15] If $C_{m}(R), C_{n}(S)$ and $C_{m n}(T)$ are circulant graphs such that $C_{m}(R) \square C_{n}(S) \cong C_{m n}(T)$, then we say that $C_{m}(R)$ and $C_{n}(S)$ are divisors or factors of $C_{m n}(T)$.

Thus for any connected circulant graph, the graph and $C_{1}()=K_{1}$ are always divisors and so we call them as improper divisors of the circulant graph. Divisors which are integer multiple of improper divisors also be called as improper divisors of the circulant graph. This doesn't arise since we consider divisors of connected graphs only. Divisor(s) other than improper divisors is called proper divisor(s) of the circulant graph.

Definition 4.8. [15] A circulant graph whose only divisors are improper is called a prime circulant graph. Other circulant graphs are called composite circulant graphs.

Theorem 4.9. [15] [Factorization Theorem On Circulant Graphs ]

Let $m$ and $n$ be relatively prime integers. If $R \subseteq\left[1, \frac{m}{2}\right]$, $S$ $\subseteq\left[1, \frac{n}{2}\right]$ and $T \subseteq\left[1, \frac{m n}{2}\right]$ with $T=d n R \cup d m S$ for somed such that $\operatorname{gcd}(m n, d)=1$, then $C_{m n}(T) \cong C_{m}(R) \square C_{n}(S)$. $\square$

Theorem 4.10. [15] If $n \neq 4$ and $1 \in R$, then $C_{n}(R)$ is a prime circulant.

Corollary 4.11. [15] If $n \neq 4$ and $R$ contains an integer relatively prime to $n$, then $C_{n}(R)$ is prime circulant. $\square$

Corollary 4.12. [15] If $n$ is a prime power other than 4 and $C_{n}(R)$ is connected, then $C_{n}(R)$ is prime circulant for all $R \neq \phi$. $\square$

\section{Theorem 4.13. [15] [Fundamental Theorem of Circu-} lant Graphs ]

Every connected circulant graph is the unique product of prime circulant graphs (uniqueness up to isomorphism).

Remark 4.14. $[13,15]$ If $G$ is a connected graph such that $G \cong G_{1} \square G_{2} \square \ldots \square G_{k}$, then the diameter of $G$, $\operatorname{dia}(G)=\sum_{i=1}^{k} \operatorname{dia}\left(G_{i}\right)$. 
Thus, we can find the diameter of any given circulant graph, provided diameters of its prime circulant graphs are known. Also the above relation helps to generate (circulant) graphs of bigger diameters.

\section{Remark 4.15. [15]}

1. In prime factorization of connected circulants $C_{1}()$ $=K_{1}$ and $C_{2}=P_{2}$ act similar to 1 and 2 among the set of all natural numbers, respectively. Thus, $C_{1}($ ) is a unit, like 1 in number theory.

2. There exist two types of prime circulant graphs of order $n$, one with periodic cycle(s) of length $n$ and the other without periodic cycle of length $n$.

3. The theory of factorization of circulants is similar to the theory of factorization of natural numbers and one of the very few well-known mathematical structures so vividly classified (expressed) in terms of prime factors. It can be applied in cryptography.

4. We developed VB programs POLY215.exe and POLY315.exe to show visually how the transformations $\Theta_{n, r, t}$ and $\Phi_{m, n}$ act on $C_{n}$ and $C_{m} \square C_{n}$,respectively for different values of $m$ and $n, m, n \in \mathbb{N}$.

5. An interesting problem is, for a given integer $n$, finding the number of prime (composite) circulant graphs of order either equal to $n$ or less than or equal to $n$.

6. One can develop theories similar to the theory of Cartesian product and factorization of circulant graphs to the other standard products of circulant graphs.

Conclusion This study covers a few isomorphic properties of circulant graphs. One can go for a similar study on Cayley graphs.

Acknowledgment The author expresses his sincere thanks to Prof. Lowell W Beineke, Indiana-Purdue University, U.S.A., Prof. Brian Alspach, University of Newcastle, Australia, Prof. M.I. Jinnah, University of Kerala, Trivandrum, India and Prof. V. Mohan, Thiyagarayar College of Engineering, Madurai, Tamil $\mathrm{Nadu}$, India for their valuable suggestions and guidance and Dr. A. Christopher, Dr. P. Wilson and Mr. R. Satheesh of S.T. Hindu College, Nagercoil, India for their assistance to develop the VB programs to show how the transformations defined on circulant graphs to obtain their isomorphic graphs is taking place. He also expresses his gratitude to Central University of Kerala (CU Kerala), Periye - 671 316, Kasaragod, Kerala, India and St. Jude's College, Thoothoor - 629 176,
Kanyakumari District, Tamil Nadu, India for providing facilities and CU Kerala and Lerroy Wilson Foundation, India (www.WillFoundation.co.in) for providing financial assistance to do this research work.

\section{References}

1. V. Vilfred, "A few properties of circulant graphs: Selfcomplementary, isomorphism, Cartesian product and factorization" in $7^{\text {th }}$ International Conference on Modeling, Simulation and Applied Optimization (ICMSAO2017), American University of Sharjah, Sharjah, April 2017.

2. P. J. Davis, Circulant Matrices, Wiley, New York, 1979.

3. A. Adam, "Research problem 2-10", J. Combinatorial Theory, 3, 393, 1967.

4. B. Alspach, J. Morris and V. Vilfred, "Self-complementary circulant graphs", Ars Com., 53, 187-191, 1999.

5. B. Alspach and T. B. Parsons, "Isomorphism of circulant graphs and digraphs", Discrete Math., 25, 97-108, 1979.

6. R. Arratia, A. D. Barbour and Simon Tavare, "Random Combinatorial Structures and Prime Factorizations", A.M.S.Notices, 44, 903-910, 1997.

7. F. T. Boesch and R. Tindell, "Circulant and their connectivities", J. Graph Theory, 8, 487-499, 1984.

8. B. Elspas and J. Turner, "Graphs with circulant adjacency matrices", J. Combinatorial Theory, 9, 297-307, 1970.

9. F. Harary, Graph Theory, Addison-Wesley, Reading, M.A., 1969.

10. W. Imrich and H. Izbicki, "Associative products of graphs", Monatsh. Math., 80, 277-281, 1975.

11. I. Kra and S. Simanca, "On Circulant Matrices", AMS Notices, 59 (3), 368-377, 2012.

12. C. H. Li, "On isomorphisms of finite Cayley graphs-a survey", Discrete Math. 256, 301-334, 2002.

13. G. Sabidussi, "Graph multiplication", Math. Z., 72, 446457, 1960.

14. H. Sachs, "Uber selbstkomplementare Graphen", Publ. Math. Debrecen, 9, 270-288, 1962.

15. V. Vilfred, "A Theory of Cartesian Product and Factorization of Circulant Graphs", Hindawi Pub. Corp. - J. Discrete Math., Vol. 2013, Article ID 163740, 10 pages.

16. V. Vilfred, "New Abelian Groups from Isomorphism of Circulant Graphs", Proce. of Inter. Conf. on Applied Math. and Theoretical Computer Science, St.Xaviers Catholic Engineering College, Nagercoil, Tamil Nadu, India, xiii-xvi, 2013.

17. V. Vilfred, " $\sum$-labelled Graphs and Circulant Graphs", Ph.D. Thesis, University of Kerala, Trivandrum, India, 1994.

18. V. Vilfred and P. Wilson, "New Families of Circulant graphs without Cayley Isomorphism Property with $m_{i}=5$ ", Int. Journal of Scientific and Innovative Mathematical Research, 3 (6), 39-47, 2015.

19. V. Vilfred and P. Wilson, "New Family of Circulant Graphs without Cayley Isomorphism Property with $m_{i}=7$ ", IOSR Journal of Mathematics, 12, 32-37, 2016.

20. H. Zhang, "Self-complementary symmetric graphs", J. Graph Theory, 16, 1-5, 1992. 